\title{
Perform the Regulatory Process: A National Approach for Evaluation of the Pre-Hospital Emergency Information System in I.R. Iran
}

\author{
Somayeh ABEDIAN ${ }^{a, b, 1}$, Pirhossein KOLIVAND ${ }^{a}$, Hamid Reza LORNEJAD ${ }^{\mathrm{a}}$ \\ ${ }^{a}$ National Emergency Medical Services Organization, Ministry of Health and Medical \\ Education, I.R. Iran \\ ${ }^{\mathrm{b}}$ Department of Information Technology Management, Islamic Azad University, Qazvin, Iran
}

\begin{abstract}
Evaluation of Emergency Medical Services Management Information System (EMSIS) makes it possible to assess the extent to which the objectives of supporting of healthcare delivery services. This paper presents an overview of the regulatory process in prehospital EMS electronic data registration and provides a minimum data set for the purpose of developing such a care system on a national scale. It further offers an evaluation framework for such systems.
\end{abstract}

Keywords. Pre-Hospital Emergency Medical Services Management Information System (EMSIS), operations leadership center (OLC), Key performance indicators, Pre-Hospital Care Report (PCR)

\section{Introduction}

Prehospital care is provided by Emergency Medical Services (EMS) organization staff, the initial health care providers at the disaster scene [1]. EMS personnel are often the first to recognize the nature of a disaster, immediately evaluate the situation, determine the need for resources and perform emergency medical procedures [2]. The Emergency Medical Services Management Information System (EMSIS) has been developed with the aim of improving the follow-up process, monitoring the calls for first aid procedures of pre-hospital emergency (phone number: 115 in Iran). In this system, the information of a mission is recorded from the moment of receiving a phone call and all information received from the caller by the call center operators (trained nurses) is added to the mission Electronic Medical Record (EMR) in order to meet the information needs and support the delivery of emergency medical services [3]. In case of the need to send a rescue vehicle such as a motorlance, ambulance or helicopter, this operation is performed by the Operations Leadership Center (OLC) and dispatch information is also registered in the patient's EMR. As soon as the paramedics or Emergency Medical Technicians (EMTs) arrive at the patient bedside, the information of the patient observations and vital signs are added to the mission information. If the EMT needs any advice from specialists present at OLC (under the supervision of National EMS organization), their diagnosis and prescribed item are added to the mission information. In addition, all telephone calls of a mission are recorded for future reference (for example, in case of legal proceedings or complaints) $[3,4]$. After transferring patient to the hospital and hospitalization, all the information about administration data or medical procedures performed for the patient in the hospital are registered in the mission EMR. To perform the whole EMS processes through a comprehensive information system, the existence of special capabilities and

\footnotetext{
${ }^{1}$ Corresponding and first author: Somayeh Abedian, Ministry of Health and Medical Education, Tehran, Iran,

E-mail: S.abedian@behdasht.gov.ir, So.abedian@gmail.com
} 
key indicators is crucial [5]. Therefore, the Iranian EMS organization and the Statistics and Information Technology Management Center of the Ministry of Health and Medical Education (ITMC-MOHME) determined and developed the main indicators of a capable system to meet EMS requirements. Due to the vitality of providing pre-hospital services and direct link with human lives, the high efficiency of this system in both technical and functional dimensions was studied. This paper provides the definitions of a number of Key Performance Indicators (KPI) to assess the quality, functionality and reliability of such a comprehensive system.

\section{Methods}

In this project, a model for evaluation of Emergency Medical Services Management Information Systems (EMSIS) was developed. The model contains a number of indicators in two categories that evaluate in line with weight-based decision tree framework [6,7]. In order to identify and classify the indicators, help was enlisted from emergency medicine specialists and experts in EMS affairs alongside health informatics professionals, IT experts and other involved people [9]. Also, the collaboration of ISO corresponding technical committees in Health informatics (ISO/TC 215) members was utilized. EMSIS is divided into different parts. According to experts, the categories fall in two groups of Functional and Technical, each supporting some primary sub-groups. More than 25 separate parts with unique features were defined. After the first round of consulting experts, they were decreased to 10 subcategories, and then final KPIs were reallocated. Thus, in this model attempts were made to combine all similar features and define some categories to support all KPIs. The criteria identified in the framework were given expert ratings. In other words, they weighted to qualitative indicators were converted to quantitative and metric indices. Then, the system was assessed in terms of supporting the functional criteria.

\section{Results}

\subsection{Evaluation components}

The indicators are used for the government to guarantee the reliability, responsibility and accessibility of this critical information system. The system is a live operational information system at the level of Transaction Processing Systems (TPS), and uninterrupted activity is inevitable [5]. The first step constituted determining clear objectives for the system. For every goal, sub-groups were defined under functional or technical groups. The framework includes more than 160 criteria assigned to 10 categories in two main branch of functional and technical components. The technical components are typically adapted with the infrastructure of software and technical support. The functional components are related to the operational requirements necessary to be registered electronically. These components are not specific to parts of the procedure. They may be utilizing in different parts of the phone-calls to discharge patient from EMS. For instance, vital signs that are recorded in EMT's part in a cellphone application will be used by physicians at medical advice department to observe the patient's clinical condition and prescribe medicines or a treatment course. In the technical aspect, the security of system has to be observed in all sub-systems. The functional components include the following:

1. Telephone triage support,

2. Electronic registration of all dispatch and mission information, 
3. Medical Advice Unit Support,

4. Ability to record admission unit information (pre-hospital and hospital),

5. Support for electronic and automatic recording of clinical and non-clinical data by EMT or EMS workers (completion of the PCR electronic form and other critical data at the patient's bedside).

The functional indicators include more than 130 items of which a few of Medical Advice Unit Support criteria are shown in Table 1.

Table 1. A number of key performance indicators (KPI) for the evaluation of Medical Advice Unit Support (Functional type)

\begin{tabular}{lc}
\multicolumn{1}{c}{ Indicator(s) } & Weight in category \\
\hline $\begin{array}{l}\text { Fields to insert the doctor's diagnosis in the form of checkbox menus or } \\
\text { optional and free-text field for descriptions }\end{array}$ & 5 \\
\hline $\begin{array}{l}\text { Ability to automatically register and attach all conversations with the } \\
\text { consulting physician to EMR }\end{array}$ & 8 \\
\hline Text fields to register suspicious cases & 4 \\
\hline $\begin{array}{l}\text { The possibility to attach a new medical record to the original record with } \\
\text { the insertion of time (connection to last medical record) in case of re- } \\
\text { consultation for a patient and opening a new consultation form }\end{array}$ & 4 \\
\hline $\begin{array}{l}\text { Visibility of the number of EMTs and callers in the waiting queue with } \\
\text { separate alarms (mechanized, audible, colored, etc.) for the physician }\end{array}$ & 8 \\
\hline
\end{tabular}

The technical components include the following:

6. Security considerations (according to security certification from ICT Ministry)

7. Technical performance

8. Standard documentation of software

9. System performance and efficiency

10. Software flexibility

Technical type encompasses more than 35 indicators of which a few standard documentation criteria of software category are presented in Table 2.

Table 2. A number of key performance indicators (KPI) for evaluation of standard documentation of software category (Technical aspect)

\begin{tabular}{lc}
\multicolumn{1}{c}{ Indicator(s) } & Weight in category \\
\hline Standard software development document & 4 \\
A competency certificate issuance from EMS organization & 4 \\
Ability to export dynamic reports as Excel, Microsoft Word, XML, & 3 \\
etc. & \multicolumn{2}{|c}{2} \\
Software roadmap document & 3 \\
Software application document & 3 \\
Software architecture document & 3 \\
Software data model and database design document & 3 \\
Existing process models and roles document & 3 \\
Provision of educational documentation depending on user levels & 3 \\
Data dictionary document & 3 \\
\hline
\end{tabular}

\subsection{Indicator weighting}

In this model, each component comprises some main subgroups and also their subsidiary subgroups. Functional and technical component each include 5 subsets. The functional and technical components have more than 130 and 35 criteria, respectively. The criteria were weighted according to experts' comments, and after finalization, were assessed by private companies developing EMSIS product based on published criteria via a selfevaluation process. Finally, a some time is allocated to each company to present their product and defend its potentials and capability of their system within the context. Finally, based on the evaluation results, a national certificate was issued to represent 
capability level of the software. This certificate indicates a product's quantitative ranking in two categories and ten subgroups. The quantitative results of the certification involve three levels which are shown in Table 3.

Table 3. Status of evaluation results in sub-groups

\begin{tabular}{lll}
\hline \multicolumn{1}{c}{ Status } & \multicolumn{1}{c}{ Description } & \multicolumn{1}{c}{ Score } \\
\hline Optimal & In this status, the system ideally meets the requirements. & Above $80 \%$ \\
\hline acceptable & $\begin{array}{l}\text { In this status, the system satisfies minimum acceptable } \\
\text { functionality and the software needs further development. }\end{array}$ & Between 60 and $80 \%$ \\
\hline Rejected & $\begin{array}{l}\text { This level is rejected and the technical and functional } \\
\text { capabilities are not achieved. }\end{array}$ & Less than $60 \%$ \\
& & \\
\hline
\end{tabular}

\section{Discussion}

An assessment framework is designed, implemented and would annually improve the criteria based on professional advice and emerging requirements. To this aim, a committee consisting of all relevant experts was formed. In this process, sponsored by the National EMS organization, ITMC-MOHME, Computer Union Organization (NonGovernmental) and Supreme Council of Cyberspace (under presidential supervision), attempts were made to improve the capabilities of EMSIS based on national and international standards. This paper seeks to serve the function of evaluating and achieving more efficient systems, effective KPIs in service delivery (aspects evaluation) and developing evaluation methods for such systems. Moreover, the first purpose of this work was improving the quality of EMS services in response time, acceptability, continuity/sustainability, patient safety, clinical effectiveness and welfare for citizens. In this regard, a set of criteria and indicators have been published, according to which relevant software is assessed, and certificates are issued for each product in ten classifications.

\section{References}

[1] Washington DC. Committee on Guidance for Establishing Crisis Standards of Care for Use in Disaster Situations, Institute of Medicine. Crisis Standards of Care: A Systems Framework for Catastrophic Disaster Response. Washington (DC): National Academies Press (US). DC: National Academies Press; 2012.

[2] Marks MR, Bowers C, DePesa NS, Trachik B, Deavers FE, James NT. REACT: A paraprofessional training program for first responders-A pilot study. Bulletin of the Menninger Clinic. 2017 Jun;81(2):150-66.

[3] Ramadanov N, Klein R, Ramadanova N, Behringer W. Influence of Time of Mission on Correct Diagnosis by the Prehospital Emergency Physician: A Retrospective Study. Emergency Medicine $\begin{array}{llllll}\text { International } & \text { [Internet]. } & 2019 & \text { Apr } & 2 ; 2019 & \text { Available }\end{array}$ https://www.hindawi.com/journals/emi/2019/3727081.

[4] EMS1 Academy. EMS protocols all paramedics should follow [Internet]. https://www.ems1.com. 2019 [cited 2019 Aug 16]. Available from: https://www.ems1.com/ems-education/articles/ems-protocols-allparamedics-should-follow-7c1zyNEC6v1SjDQM/.

[5] Pap R, Lockwood C, Stephenson M, Simpson P. Indicators to measure prehospital care quality: a scoping review. JBI Database of Systematic Reviews and Implementation Reports. 2018 Nov;16(11):2192-223.

[6] Doğan SZ, Arditi D, Murat Günaydin H. Using decision trees for determining attribute weights in a casebased model of early cost prediction. Journal of Construction Engineering and Management. 2008 Feb;134(2):146-52.

[7] Mark H. A Decision Tree-Based Attribute Weighting Filter for Naive Bayes. Research and Development in Intelligent Systems XXIII. New Zealand: Springer; 2007;59-70.

[8] Kass GV. An Exploratory Technique for Investigating Large Quantities of Categorical Data. Journal of the Royal Statistical Society. 1980;29(2):119-27.

[9] McSherry D. Strategic induction of decision trees. Knowledge-Based Systems. 1990;12(5-6):269-75. 